\title{
Resveratrol, an activator of SIRT1, upregulates AMPK and improves cardiac function in heart failure
}

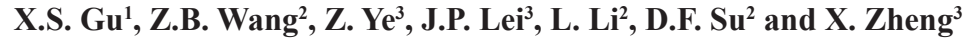 \\ ${ }^{1}$ Department of Cardiology, \\ The Second Affiliated Hospital of Soochow University, Suzhou, China \\ ${ }^{2}$ Department of Pharmacology, College of Pharmacy, \\ Second Military Medical University, Shanghai, China \\ ${ }^{3}$ Department of Cardiology, Changhai Hospital, \\ Second Military Medical University, Shanghai, China \\ Corresponding authors: X. Zheng \\ E-mail: zhengxing57530@163.com
}

Genet. Mol. Res. 13 (1): 323-335 (2014)

Received April 25, 2013

Accepted August 22, 2013

Published January 17, 2014

DOI http://dx.doi.org/10.4238/2014.January.17.17

\begin{abstract}
Reduced AMP-activated protein kinase (AMPK) expression has been shown to play a significant role in the cardiac dysfunction in heart failure. This study was designed to examine the effect of resveratrol, a potent activator of silent information regulator (SIRT1), on cardiac function and AMPK expression in heart failure. Adult male rat left anterior descending arteries were ligated, and they were fed with either a regular diet or a diet enriched with resveratrol. Heart failure was produced by myocardial infarction, and was associated with markedly increased AMPK and SIRT1 protein levels. Resveratrol treatment had a tremendous beneficial effect, both in terms of improving AMPK expression and on cardiac function. Decreased cardiac function and AMPK expression were also found in SIRT1 knockout (+/-) mice. In cultured cardiomyocytes, resveratrol increased AMPK and SIRT1 expressions, and overexpression of SIRT1 was found to be sufficient to activate AMPK in $\mathrm{H} 9 \mathrm{c} 2$ cells. In contrast, pretreatment
\end{abstract}


of cardiomyocytes with an SIRT1 antagonist, nicotinamide, blocked these beneficial effects of resveratrol. Therefore, the protective effects of resveratrol were found to be dependent on its ability to activate SIRT1 and improve AMPK expression. These results demonstrated that in heart failure, the enzymatic activity of cardiac SIRT1 is increased, which contributes to increased expression of AMPK, and resveratrol enhances the expression of AMPK and improves cardiac function through the activation of SIRT1.

Key words: Heart failure; SIRT1; AMPK; Resveratrol

\section{INTRODUCTION}

Heart failure is a progressive muscular disorder leading to deterioration of the heart function characterized by contractile dysfunction and chronic energy deficit. In consequence, the failing heart is unable to meet the normal metabolic and energy needs of the body. The transition between compensated left ventricular hypertrophy and de-compensated heart failure is multifactorial, although metabolic disturbances are considered to play a significant role. In this respect, the AMP-activated protein kinase (AMPK) could be a potential target in heart failure development. AMPK senses the energy state of the cell and orchestrates a global metabolic response to energy deprivation. AMPK is a highly conserved serine/threonine kinase that is activated by an increase of the cellular AMP-to-ATP ratio, and is considered to be a sensor of cellular energy status (Kim and Lee, 2005; Ramamurthy and Ronnett, 2006). Upon activation, AMPK inhibits ATP-consuming pathways and stimulates ATP-generating pathways to restore the cellular energy balance. AMPK is expressed in the heart and is involved in the regulation of heart failure (Turdi et al., 2010; Beauloye et al., 2011; Zaha and Young, 2012). Previous studies have shown that activation of AMPK prevents the progression of heart failure.

Resveratrol, a natural phytoalexin found in red grape skins, red wine, and peanuts, has been reported to exert a variety of biological effects. Through its antioxidative properties, it has been shown to prevent or slow the progression of a wide variety of illnesses, including cardiovascular diseases, brain disorders, and cancer (Hung et al., 2000; Cho et al., 2012; Shin et al., 2012). In short-term type 1 diabetes, resveratrol was shown to improve cardiac function after ischemia-reperfusion injury by enhancing the anti-oxidative capacity of the heart (Thirunavukkarasu et al., 2007). All of these resveratrol effects appear to be related to its ability to induce silent information regulator ( $\operatorname{Sir} 2 \alpha$, also known as SIRT1) activity (Sulaiman et al., 2010). SIRT1 is the founding member of a large family of class III histone deacetylases, and is a redox-sensitive enzyme that needs cellular NAD as a cofactor for its deacetylation reactivity (Imai et al., 2000). In mice, SIRT1 is highly expressed in the embryonic heart, and its knockout results in cardiac developmental defects (Cheng et al., 2003). Its anti-apoptosis properties have been demonstrated in cardiomyocytes, in which SIRT1 overexpression protected cells against oxidative damage, whereas SIRT1 inhibition promoted cell death (Alcendor et al., 2007). Another study showed that the SIRT1 activator SRT1720 increased AMPK activity, whereas SIRT1-specific inhibitors decreased AMPK activity in adipocytes (Jiang et al., 2012).

The aim of the present study was to test whether SIRT1 is a critical regulator of AMPK signaling in heart failure. Our results showed that resveratrol, through activation of SIRT1 and AMPK, improved cardiac function in heart failure rats. We also showed reduced cardiac 
function and AMPK expression in SIRT1 (+/-) knockout mice. Additionally, we showed that SIRT1 acts as a transcriptional activator of AMPK gene expression in SIRT1-overexpressed H9c2 cells. These results demonstrate, for the first time, that resveratrol, acting via SIRT1, could regulate AMPK expression in $\mathrm{H} 9 \mathrm{c} 2$ cells, and that this effect could explain the mechanism underlying the therapeutic effect of resveratrol on heart failure.

\section{MATERIAL AND METHODS}

\section{Animals}

All animals received humane care in compliance with the Principles of Laboratory Animal Care formulated by the National Society for Medical Research, and the Guide for the Care and Use of Laboratory Animals prepared by the National Academy of Sciences and published by the National Institutes of Health (NIH Publication No. 86-23, revised in 1996). The use of animals in our experiments was approved by the Institutional Animal Care and Use Committee of the Second Military Medical University, Shanghai. We used male Sprague-Dawley rats 8 weeks of age. The animals were anesthetized with a solution of $80 \mathrm{mg} / \mathrm{kg}$ katamine hydrochloride $+12 \mathrm{mg} / \mathrm{kg}$ xylazine. After intubation, the animals received mechanical ventilation with a Harvard ventilator ( $90 \mathrm{~m} / \mathrm{min}$ frequency; $2.0 \mathrm{~mL}$ tidal volume) and a standard limb lead II ECG was recorded by an ECG analysis system (SL Power Electronics, Shanghai). A left thoracotomy was performed in the intercostal space of the apex beat, and the heart was quickly exposed. The anterior interventricular branch of the left coronary artery was identified and ligated between the edges of the left auricular appendage and the pulmonary artery with 6-0-prolene sutures. The lungs were hyperinflated, and the chest wall was closed with a previously performed purse-string suture. For the sham operation, we used the same surgical procedure, but without the disruption of the pericardium and the ligation of the left anterior descending (LAD) coronary artery. After the coronary artery ligation, the animals were confined to the vivarium for 1 week, and an echocardiogram was performed to confirm the presence of infarction. Subsequently, rats were randomly divided into the following three main groups: the resveratrol group, the heart failure group, and the control group. The resveratrol group was fed $2.5 \mathrm{mg} / \mathrm{kg}$ resveratrol (Sigma, St. Louis, MO, USA) per day (Wojciechowski et al., 2010) for 16 weeks. The control and heart failure groups were fed a regular diet without resveratrol. A total of 10 or 11 animals comprised each group. All animals had free access to water and food and received daily bedding changes. The knockout SIRT1 (+/-) mice (CD1 strain) were bred in our laboratory, and only male mice were used in the study.

\section{Echocardiography}

Rats were anaesthetized with controlled inhalation of a mixture of $1.5 \%$ isoflurane and $0.5 \mathrm{~L} / \mathrm{min}$ oxygen, which has been shown to have only a minimal cardiac depressive effect (Rojas et al., 2008). Animals were placed on a thermally controlled foam pad to maintain body temperature between $35^{\circ}$ and $38^{\circ} \mathrm{C}$ throughout the recordings. Transthoracic M-mode echocardiography was performed with a Siemens ACUSON Sequoia 256 imaging system (Siemens) using a 13-MHz broadband transducer and an echocardiography coupling gel to maximize contact between the transducer and the chest. M-mode recordings of the left ventricle (LV) were obtained in the two-dimensional parasternal long-axis and short-axis imaging plane 
in accordance with the American Society of Echocardiography, using the level of the papillary muscle as a guide. All images were recorded at the lowest depth setting and sector width possible. Images were digitally recorded on a magneto-optical disk. Left ventricle end-diastolic diameter (LVEDd) and end-systolic diameter (LVESd) were measured off-line according to standard procedures by an experienced echocardiographer who was blind to the experimental protocol. Three consecutive cardiac cycles were measured and averaged to minimize the effects of noise and respiratory variation. The mean fractional shortening (FS) percentage was calculated with a widely used formula: FS (\%) $=$ [(LVEDd - LVESd) / LVEDd $] 100$.

\section{Determination of plasma B-type natriuretic peptide (BNP)}

Blood samples were collected into Lavender Vacutainer tubes containing EDTA and 0.6 trypsin inhibitor $\mathrm{U} / \mathrm{mL}$ blood aprotinin, and were centrifuged at $1600 \mathrm{~g}$ for $15 \mathrm{~min}$ at $4^{\circ} \mathrm{C}$ to separate plasma. Supernatants were collected and stored at $-70^{\circ} \mathrm{C}$. Plasma BNP levels were measured by a biotin-coupled anti-BNP antibody/streptavidin solid-phase chromatographic immunoassay with the Status First ${ }^{\mathrm{TM}}$ CHF BNP test device. The functional sensitivity of the assay was the lowest BNP concentration that could be reproducibly measured with a maximum total coefficient of variation of $20 \%$, which was determined as $20 \mathrm{pg} / \mathrm{mL}$.

\section{Langendorff heart preparation}

Mice were killed by cervical dislocation. The heart was rapidly excised and placed in ice-cold modified Krebs-Henseleit (KH) buffer (118 mM NaCl, $4.7 \mathrm{mM} \mathrm{KCl}, 1.2 \mathrm{mM} \mathrm{MgSO}$, $1.2 \mathrm{mM} \mathrm{KH}_{2} \mathrm{PO}_{4}, 1.2 \mathrm{mM} \mathrm{CaCl}_{2}, 11 \mathrm{mM}$ d-glucose, $25 \mathrm{mM} \mathrm{NaHCO}_{3}$, and $2 \mathrm{mM}$ pyruvic acid) with $95 \% \mathrm{O}_{2}$ and $5 \% \mathrm{CO}_{2}$. The aorta was immediately cannulated with a $19-\mathrm{G}$ needle (with a small circumferential grove close to the blunt tip) and perfused with gassed KH buffer, according to Langendorff, at a constant perfusion pressure of $80 \mathrm{mmHg}$. Left ventricular systolic pressure (LVSP) was measured with a water-filled balloon (made of domestic food wrap) connected to a disposable pressure transducer (Braun, Melsungen, Germany). The left atrium was removed, and the balloon was inserted into the left ventricle. Left ventricular end-diastolic pressure was set at $3-5 \mathrm{mmHg}$ by adjusting the balloon volume. After a stabilization period of 10-15 min, baseline values of LVSP were obtained. Next, 100- $\mu \mathrm{L}$ bolus injections of modified $\mathrm{KH}$ buffer were applied three times to determine injection-induced changes in LVSP (Wang et al., 2002). The following parameters were recorded: heart rate (HR, bpm), systolic pressure $(\mathrm{mmHg}),+\mathrm{dP}^{-\mathrm{dt}_{\max }}$, and $-\mathrm{dP}^{\mathrm{d}} \mathrm{dt}_{\text {max }}(\mathrm{mmHg} / \mathrm{s})$.

\section{Preparation of protein lysate}

For Western blot analysis, $50 \mathrm{mg}$ frozen heart tissue was homogenized in $1.5 \mathrm{~mL}$ lysis buffer (20 mM HEPES, pH 7.4, 2 mM EGTA, $1 \mathrm{mM}$ dithiothreitol, $1 \mathrm{mM}$ sodium orthovanadate, $1 \%$ Triton X-100, 10\% glycerol, $2 \mu \mathrm{M}$ leupeptin, $1 \mathrm{mM}$ 4-(2-aminoethyl) benzenesulfonyl fluoride hydrochloride, $1 \mathrm{mM}$ aprotinin, and $400 \mu \mathrm{M}$ phenylmethylsulfonyl fluoride) at $8000 \mathrm{rpm}$. The homogenate was centrifuged at $0^{\circ} \mathrm{C}$ and $14,000 \mathrm{~g}$, the supernatant was collected, and the protein concentration was determined by the Bradford assay. Aliquots of tissue lysate were stored at $-80^{\circ} \mathrm{C}$ (Pacholec et al., 2010). 


\section{Western blot analysis}

Proteins $(50 \mu \mathrm{g})$ were resolved by $10 \%$ sodium dodecyl sulfate-polyacrylamide gel electrophoresis and transferred to a polyvinylidene difluoride membrane. Membranes were blocked with $10 \%$ milk and hybridized overnight at $4{ }^{\circ} \mathrm{C}$ with anti-AMPK $(1: 1000$; Santa Cruz), anti-SIRT1 (1:1000; Abcam), and anti-GAPDH (1:1000; Cell Signal) antibodies. Membranes were washed with $1 \mathrm{X}$ phosphate-buffered saline-Tween and hybridized with appropriate secondary antibody (1:2000) in 5\% milk. After washing, the signal was developed using a chemiluminescence reagent (Santa Cruz) and detected by autoradiography.

\section{Construction of expression plasmids}

Full-length rat SIRT1 cDNA was amplified by polymerase chain reaction from rat myocardial tissue RNA using the following primers: forward: 5'-TTCAGCAACACCTCATG ATT-3' and reverse: 5'-CAATGCTGTTTCTTCTTTGC-3'. The cDNA was cloned into pcDNA3.1 and pEGFP-N1 expression plasmids (Invitrogen) and its sequence was verified.

\section{Cell culture and transfection}

Commercially available H9c2 rat embryonic cardiac myoblasts (ATCC) were grown in Dulbecco's modified Eagle's medium (DMEM) supplemented with 10\% fetal bovine serum and $1 \%(\mathrm{v} / \mathrm{v})$ streptomycin/penicillin (Invitrogen) at $37^{\circ} \mathrm{C}$ and $5 \% \mathrm{CO}_{2}$. When cells reached about $80 \%$ confluence in appropriate culture dishes, they were seeded on $100-\mathrm{mm}$ tissue culture dishes and transfected for $24 \mathrm{~h}$ with $5 \mu \mathrm{g}$ plasmid DNA per dish using Transfectin ${ }^{\circledR}$ transfection reagent (BioRad). Briefly, $10 \mu \mathrm{L}$ Transfectin was added to $500 \mu \mathrm{L}$ serum-free DMEM followed by the addition of plasmid DNA. The mixture was incubated for $15 \mathrm{~min}$ at room temperature prior to adding it to the cells. In specific protocols, cells were also treated with different concentrations of resveratrol and nicotinamide for $24 \mathrm{~h}$.

\section{Scanning densitometry and statistical analysis}

Autoradiograms were scanned using the Scion Image for Windows analysis software, based on NIH image for Macintosh by Wayne Rasband (NIH, Bethesda, MD, USA). The signal intensity was adjusted according to the background density of the blot. Data are reported as means $\pm \mathrm{SD}$. Statistical differences among groups were determined using either the Student $t$-test for 2 groups or one-way analysis of variance (ANOVA) for more than 2 groups.

\section{RESULTS}

\section{Resveratrol treatment improved cardiac function, reduced mortality, and increased AMPK expression}

To study the effects of resveratrol in a more clinically relevant model of heart failure, the LAD artery of adult male rats was ligated, and $2.5 \mathrm{mg} / \mathrm{kg}$ resveratrol or vehicle was administered to each group daily for 16 weeks. Compared to the heart failure group, resveratrol 
treatment significantly decreased BNP levels $(391.44 \pm 132.88$ vs $876.50 \pm 164.64 \mathrm{pg} / \mathrm{mL}, \mathrm{P}<$ $0.001)$ and increased the left ventricular ejection fraction $(46.84 \pm 6.06 v s 34.44 \pm 2.13 \%, \mathrm{P}<$ 0.001) (Table 1). As shown in Figure 1, the survival rate in rats receiving resveratrol $(\mathrm{N}=11)$ was higher than that in rats treated with vehicle over 16 weeks ( 82 vs 36\%).

Table 1. Animal characteristics.

\begin{tabular}{|c|c|c|c|c|}
\hline & Control $(\mathrm{N}=10)$ & Heart failure $(\mathrm{N}=4)$ & Resveratrol $(\mathrm{N}=9)$ & ANOVA \\
\hline Body weight (g) & $457.10 \pm 37.27$ & $379.25 \pm 35.08 *$ & $408.22 \pm 34.26^{*}$ & $\mathrm{P}=0.002$ \\
\hline $\mathrm{HW} / \mathrm{BW}(\mathrm{mg} / \mathrm{g})$ & $1.95 \pm 0.15$ & $2.68 \pm 0.37^{*}$ & $2.41 \pm 0.27^{*}$ & $\mathrm{P}<0.001$ \\
\hline $\operatorname{LVEDd}(\mathrm{cm})$ & $1.03 \pm 0.13$ & $1.23 \pm 0.09 *$ & $1.21 \pm 0.09 *$ & $\mathrm{P}=0.002$ \\
\hline LVESd (cm) & $0.70 \pm 0.12$ & $1.07 \pm 0.07 *$ & $0.98 \pm 0.06^{*}$ & $\mathrm{P}<0.001$ \\
\hline LVEF (\%) & $67.49 \pm 6.88$ & $34.44 \pm 2.13^{*}$ & $46.84 \pm 6.06^{* \#}$ & $\mathrm{P}<0.001$ \\
\hline $\mathrm{BNP}(\mathrm{pg} / \mathrm{mL})$ & $105.55 \pm 54.14$ & $876.50 \pm 164.64 *$ & $391.44 \pm 132.88^{* \#}$ & $\mathrm{P}<0.001$ \\
\hline
\end{tabular}

Values are reported as means \pm SD in each group. *Significant $(\mathrm{P}<0.05)$ difference from control animals and ${ }^{*}$ heart failure group. HW/BW = heart weight to body weight ratio; LVEDd and LVESd $=$ left ventricle end-diastolic and -sistolic diameters, respectively; LVEF = left ventricular ejection fraction; BNP = B-type natriuretic peptide.
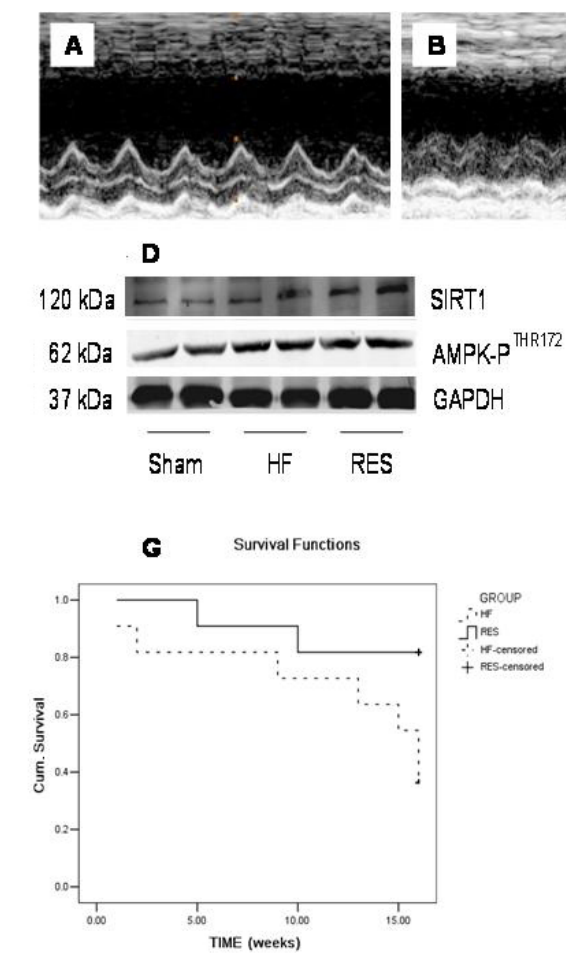

Figure 1. Survival, echocardiographic measurements and protein expression. A.-C. Representative M-mode echocardiograms of sham (A), heart failure (HF, B), and resveratrol (RES, C) groups. D. Expression of SIRT1 and AMPK. E. and F. Compared with the sham group, SIRT1 and AMPK expression significantly increased in HF $(\mathrm{P}<0.01, \mathrm{P}<0.05, \mathrm{~N}=4$, respectively) and RES $(\mathrm{N}=4, \mathrm{P}<0.01)$ groups; compared with the HF group, SIRT1 expression is higher in the RES group $(\mathrm{P}<0.01$ for both). G. Kaplan-Meier survival curves of HF $(\mathrm{N}=11)$ and RES $(\mathrm{N}=11)$ groups. Heart failure progression was associated with reduced survival rate $\left(\chi^{2}=3.98 \mathrm{P}=0.046\right)$. ${ }^{\mathrm{P}}$ $<0.01$ vs sham group, ${ }^{\Delta} \mathrm{P}<0.05$ vs sham group, ${ }^{\#} \mathrm{P}<0.01$ vs $\mathrm{HF}$ group. 
In the present study, we also investigated whether resveratrol resulted in sustained elevations in AMPK and SIRT1 expressions. Although phosphorylation of AMPK ${ }^{\text {Thr172 }}$ was significantly increased in both vehicle-treated $(\mathrm{P}<0.05)$ and resveratrol-treated $(\mathrm{P}<0.001)$ groups, resveratrol was found to significantly augment the ischemia-induced increase in $\operatorname{AMPK}^{\text {Thr172 }}(\mathrm{P}<0.01)$ compared to vehicle treatment. Similarly, SIRT1 expression was significantly increased in the resveratrol treatment group compared to the sham group $(\mathrm{P}<$ $0.05)$ and the vehicle group $(\mathrm{P}<0.05)$. These results suggest that the activation of AMPK and SIRT1 may underlie the beneficial effects of resveratrol in the myocardium and protect against ischemia-induced heart failure.

\section{Cardiac function, survival, and AMPK expression were reduced in SIRT1(+/-) mice}

Resveratrol was previously shown to improve cardiac function, reduce mortality in heart failure, and activate the SIRT1 gene (Sulaiman et al., 2010). To investigate the role of SIRT1 in resveratrol-mediated cardiac protection, SIRT1(+/-) $(\mathrm{N}=5)$ and wild-type (WT) mice $(\mathrm{N}=4)$ were subjected to Langendorff perfusion. Cardiac function was decreased in the hearts of SIRT1(+/-) mice, as evidenced by a significant decrease in HR $(231.05 \pm 16.51$ vs $286.00 \pm 16.29 \mathrm{bpm}, \mathrm{P}=0.002), \mathrm{LVP}_{\max }(111.04 \pm 13.97$ vs $141.90 \pm 6.63 \mathrm{mmHg}, \mathrm{P}=$ $0.003),+\mathrm{dP} / \mathrm{dT}_{\max }(2944.33 \pm 461.02$ vs $7122.73 \pm 1083.12 \mathrm{mmHg} / \mathrm{s}, \mathrm{P}<0.001)$, and $-\mathrm{dP} /$ $\mathrm{dT}_{\max }(2081.72 \pm 323.81$ vs $4807.48 \pm 789.79 \mathrm{mmHg} / \mathrm{s}, \mathrm{P}<0.001)$ (Table 2). Moreover, as shown in Figure 2, we observed that AMPK expression decreased in SIRT1(+/-) mice compared with WT mice $(\mathrm{P}<0.05)$. These data suggest that SIRT1 might also be an important mediator of resveratrol-mediated cardiac protection, and AMPK may be the underlying mechanism. During breeding of the SIRT1(+/-) and WT mice, we recorded the death day and found that survival time was significantly reduced in SIRT1(+/-) mice compared with WT mice $(\mathrm{P}<0.05)$.

Table 2. Cardiac parameters in Langendorff-perfused mouse hearts.

\begin{tabular}{lccc}
\hline & WT $(\mathrm{N}=4)$ & SIRT1(+/-) $(\mathrm{N}=5)$ & P value \\
\hline Heart rate $(\mathrm{bpm})$ & $286.00 \pm 16.29$ & $231.05 \pm 16.51$ & $\mathrm{P}=0.002$ \\
$\mathrm{LVP}_{\text {max }}(\mathrm{mmHg})$ & $141.90 \pm 6.63$ & $111.04 \pm 13.97$ & $\mathrm{P}=0.003$ \\
$+\mathrm{dP} / \mathrm{dT}_{\max }(\mathrm{mmHg} / \mathrm{s})$ & $7122.73 \pm 1083.12$ & $2944.33 \pm 461.02$ & $\mathrm{P}<0.001$ \\
$-\mathrm{dP} / \mathrm{dT}_{\text {max }}(\mathrm{mmHg} / \mathrm{s})$ & $4807.48 \pm 789.79$ & $2081.72 \pm 323.81$ & $\mathrm{P}<0.001$ \\
\hline
\end{tabular}

Functional parameters measured after $30 \mathrm{~min}$ of aerobic perfusion in un-paced hearts. Values are reported as means \pm SD. P value: SIRT1(+/-) $v s$ wild-type mice. $\mathrm{LVP}_{\max }=$ peak value of left ventricular systolic pressure; $+\mathrm{dP} /$ $\mathrm{dT}_{\max }=$ peak positive value of time derivative of $\mathrm{LV}$ pressure; $-\mathrm{dP} / \mathrm{dT}_{\max }=$ peak negative value of time derivative of LV pressure.

\section{SIRT1 can regulate AMPK expression in $\mathrm{H} 9 \mathrm{c} 2$ cell lines}

To further examine the role of AMPK in the effect of resveratrol on cardiac dysfunction, $\mathrm{H} 9 \mathrm{c} 2$ cells were treated with different concentrations of resveratrol for $24 \mathrm{~h}$. Western blot analysis indicated that SIRT1 expression was 4 times higher in the overexpression SIRT1 H9c2 cell line compared to normal cells (Figure 3). As shown in Figure 
4, 50 and $100 \mu \mathrm{M}$ resveratrol significantly increased AMPK and SIRT1 expressions. Our data further revealed mechanical properties that indicated that resveratrol's protective effect on cardiomyocytes was related to its effect on increasing AMPK expression. Because previous studies indicated that resveratrol was not a direct activator of SIRT1 (Pacholec et al., 2010), we constructed the overexpression SIRT1 H9c2 cell line. Nicotinamide, which can inhibit the activity of SIRT1, was treated to the overexpression SIRT1 H9c2 cell line at different concentrations for $24 \mathrm{~h}$. AMPK levels were found to increase in the overexpression SIRT1 H9c2 cell line $(\mathrm{P}<0.05)$ compared with normal cells, but decreased significantly in the 20 and $40 \mathrm{mM}$ nicotinamide treatment groups $(\mathrm{P}<0.05, \mathrm{P}<$ 0.01 , respectively). These results suggest that resveratrol can improve AMPK expression by activating SIRT1.

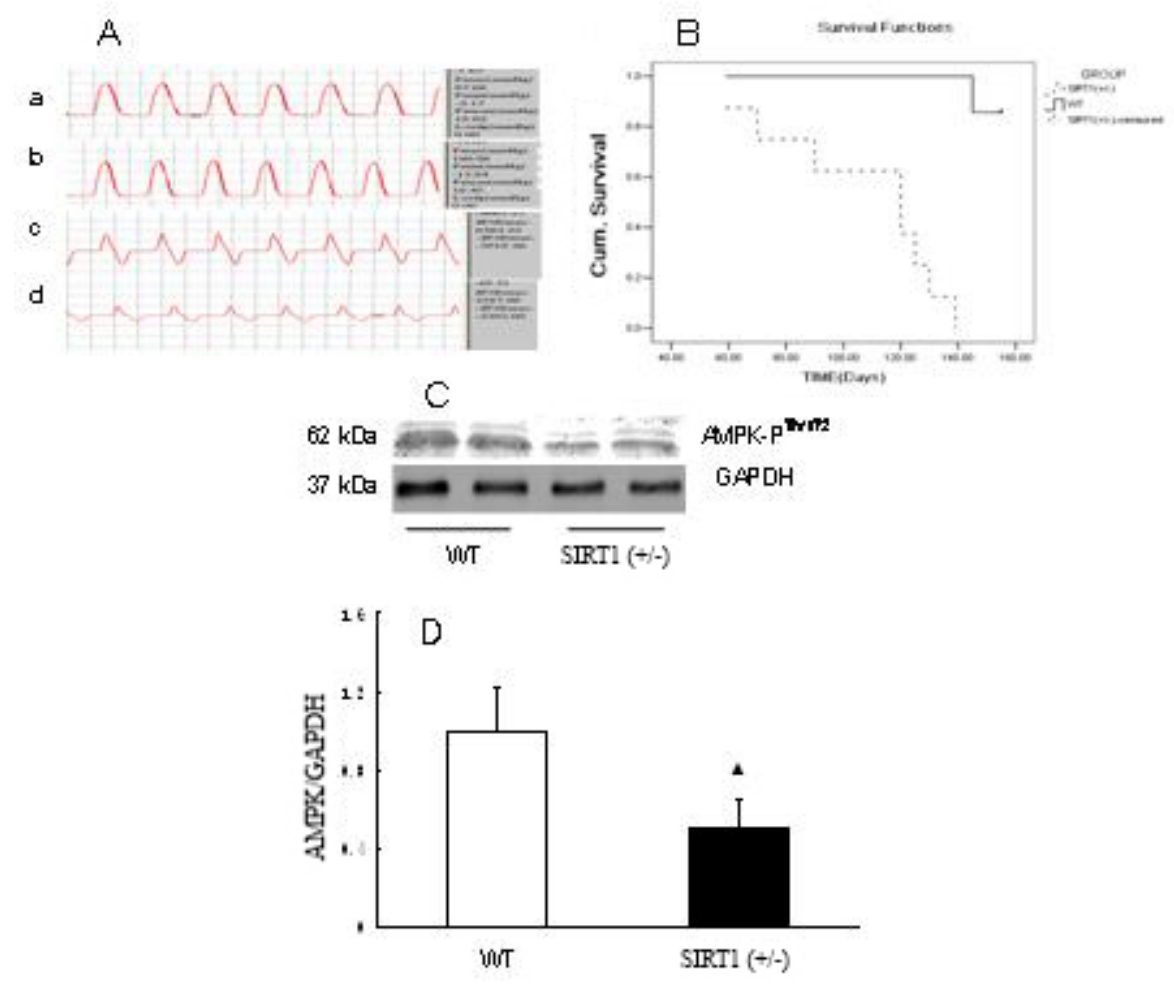

Figure 2. SIRT1(+/-) mice reduce cardiac function, lifetime and AMPK expression. HR, $\mathrm{LVP}_{\max },+\mathrm{dP}^{\mathrm{d} T} \mathrm{~T}_{\max },-\mathrm{dP} /$ $\mathrm{dT}_{\max }$ of SIRT1(+/-) $(\mathbf{A}, \mathbf{D})$ and wild-type (WT) $(\mathbf{B}, \mathbf{C})$ mice were recorded by MPA heart function analysis system (A). The measurement was done in 6 consecutive cardiac cycles, and values were then averaged. Kaplan-Meier survival curves of SIRT1(+/-) $(\mathrm{N}=8)$ and WT $(\mathrm{N}=7)$ mice were analyzed $($ B $)$. Knock-down SIRT1(+/-) was associated with reduced survival rate $\left(\chi^{2}=4.70, \mathrm{P}=0.03\right)$. AMPK expression of the cardiac tissue was obtained by Western analysis, GAPDH was used as a loading control (C). Band intensity measurements were done using Odyssey Two-Color Infrared Imaging System to semiquantitative analysis of AMPK levels. In each panel, the intensity of a given band was normalized to the intensity of the loading control (GAPDH) of the respective band and are reported as means $\pm \mathrm{SD}$ of 3 hearts analyzed in three separate experiments. AMPK levels decreased in SIRT1(+/-) compared with WT mice $(\mathrm{P}<0.05)(\mathbf{D}) .{ }^{\wedge} \mathrm{P}<0.05$ vs WT mice. 

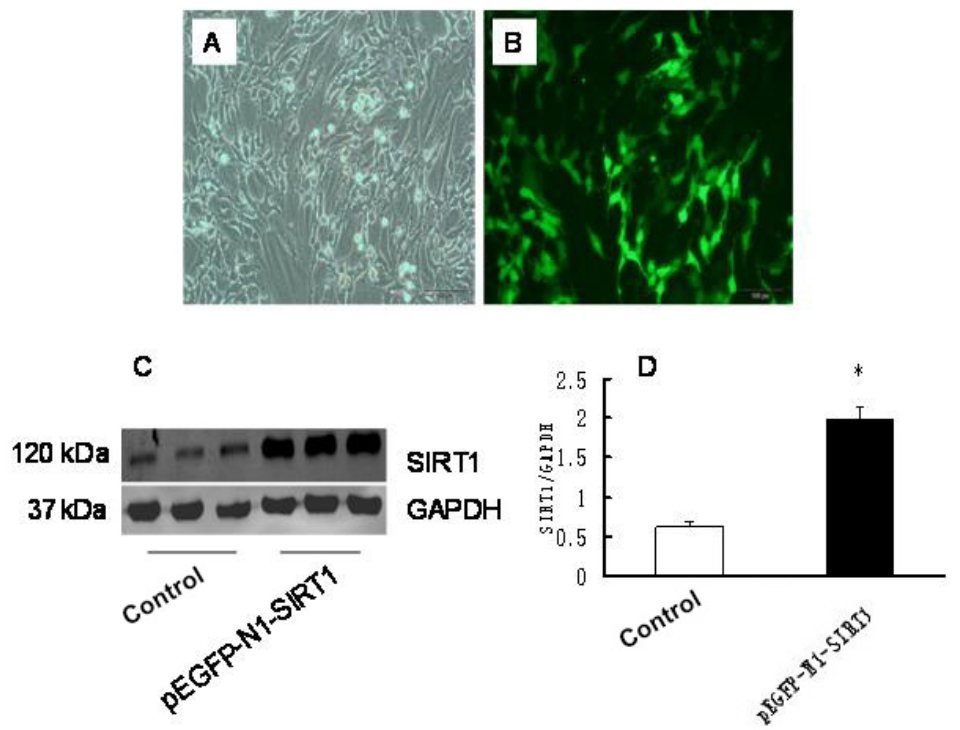

Figure 3. Construted overexpression SIRT1 H9c2 cell line. H9c2 cell transfected SIRT1 plasmid for $24 \mathrm{~h}$ (A. white light photo, B. Fluorescence photo, scar bar $=100 \mu \mathrm{m})$. SIRT1 expression of normal and overexpression SIRT1 H9c2 cell line were recorded by Western analysis $(\mathbf{C})$. Semiquantitative analysis of SIRT1 levels indicated about 4 times increasing in overexpression SIRT1 H9c2 cell line compared with normal H9c2 cell (D). ${ }^{*} \mathrm{P}<0.01$ vs control.
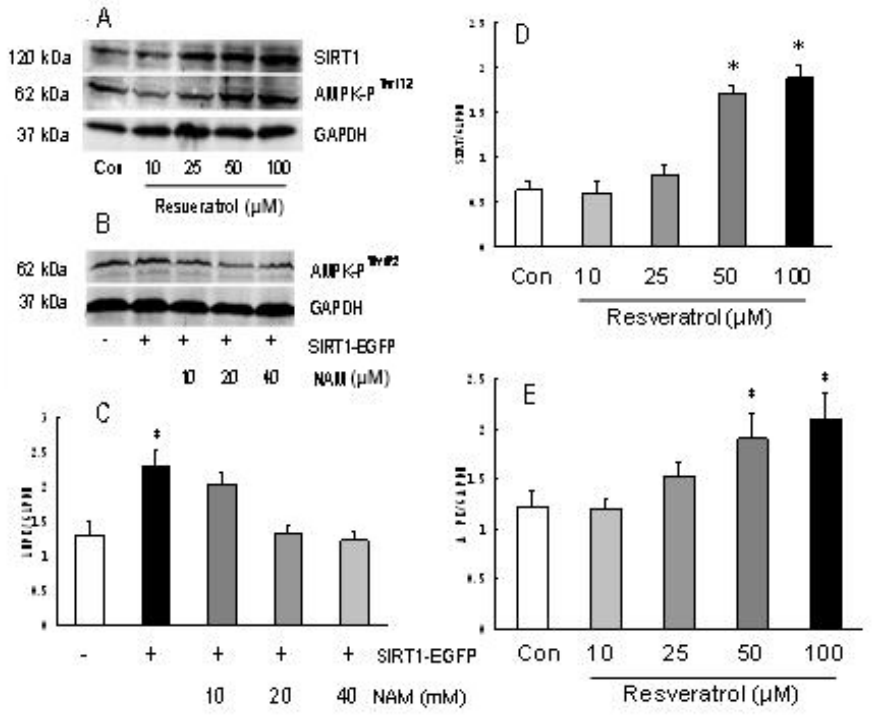

Figure 4. SIRT1 can regulate AMPK expression in H9C2 cell lines. SIRT1 and AMPK expressions of H9c2 cell under culture with different concentrations of resveratrol were obtained by Western analysis. GAPDH was used as a loading control (A). AMPK expression of overexpression SIRT1 H9c2 cell line under culture with different concentrations of nicotinamide (NAM) was obtained by Western analysis (B). AMPK expression increased in overexpression SIRT1 H9c2 cell line $(\mathrm{P}<0.01, \mathbf{C})$ compared with normal cells, but decreased in NAM $(20$ and 40 $\mathrm{mM})$ groups compared with overexpression SIRT1 H9c2 cell line $(\mathrm{P}<0.01$ for both, $\mathbf{C})$. Band intensity measurements indicate that resveratrol $(50$ and $100 \mu \mathrm{M})$ significantly increased SIRT1 $(\mathrm{P}<0.01$ for both, $\mathrm{D})$ expression and AMPK $(\mathrm{P}<0.01$ for both, $\mathbf{E}) .{ }^{*} \mathrm{P}<0.01$ vs control, ${ }^{\ddagger} \mathrm{P}<0.01$ vs overexpression SIRT1 H9c2 cell line. 


\section{DISCUSSION}

Heart failure is a prevalent cause of death in patients with cardiovascular diseases. Efforts continue to understand the molecular basis of this process and to improve or protect the heart from this complication. We provided the following lines of evidence that support a role of resveratrol and SIRT1 in the regulation of AMPK gene expression and cardiac dysfunction in heart failure: i) resveratrol could restore cardiac dysfunction, reduce mortality, and increase the expression of cardiac SIRT1 and AMPK, ii) SIRT1 and AMPK expressions increased in cardiomyocytes cultured in resveratrol, iii) SIRT1(+/-) mice demonstrated a reduction in cardiac function and low AMPK expression, and iv) overexpression of SIRT1 alone was found to be sufficient to increase the expression of AMPK, and this response was blocked by nicotinamide, an inhibitor of SIRT1.

The most important cause of heart failure is coronary artery disease and acute myocardial infarction leading to the loss of functioning myocytes, development of myocardial fibrosis, and subsequent LV remodeling, all of which contribute to the development of LV dysfunction. Therefore, in this study, we used the rat model of heart failure induced by left coronary artery ligation. Previous studies showed that daily $2.5 \mathrm{mg} / \mathrm{kg}$ body weight resveratrol treatment could reverse cardiac hypertrophy due to volume overload, limit the increase in compliance of spontaneously hypertensive rat (SHR) arteries (Behbahani et al., 2010), prevent the development of concentric hypertrophy and cardiac dysfunction in SHR rats (Thandapilly et al., 2010), and reduce infarct size and apoptotic cell death of diabetic hearts (Dekkers et al., 2008). All of the above are dangerous factors for heart failure, and suggest that $2.5 \mathrm{mg} / \mathrm{kg}$ body weight resveratrol might prevent the development of LV dysfunction, which was the oral resveratrol dose selected for this study.

This study demonstrated the novel finding that daily treatment with resveratrol arrested the development of heart failure in rats subjected to LAD ligation. Previous studies showed that resveratrol has acute antioxidant and antiinflammatory effects (Hung et al., 2000; Alcendor et al., 2007; Ghanim et al., 2011), and could also inhibit doxorubicin-induced cardiomyocyte apoptosis (Zhang et al., 2011a). Reduction of myocardial oxidative stress and apoptosis can improve cardiac function in heart failure (Lu et al., 2012). Therefore, our result that resveratrol can control the development of heart failure is reasonable. Moreover, in our study, we found that resveratrol therapy could increase the expression of SIRT1 and AMPK in failing hearts. Many studies have indicated that SIRT1 has antioxidant, anti-apoptosis, and antihypertrophy effects, indicating that increased expression of SIRT1 is likely one of the mechanisms underlying the effect of resveratrol on heart failure. Because the failing heart is unable to meet normal metabolic and energy needs of the body, and AMPK activation plays an important role in energy homeostasis and can control the development of heart failure, the activation of AMPK after resveratrol therapy appears to be another mechanism of the beneficial effect of resveratrol on heart failure. Therefore, the results of this study provide two valuable explanations for the beneficial effect of resveratrol therapy.

To evaluate the functional role of the SIRT1 gene in cardiac function, SIRT1 knockout (+/-) mice were generated. Through Langendorff perfusing, we found that SIRT1(+/-) mice had lower heart function compared with WT mice. SIRT1, an essential endogenous apoptosis inhibitor in cardiac myocytes (Alcendor et al., 2004; Zhang et al., 2011a), plays an important role in heart development. SIRT1-defective mice have shown congenital heart disease (Cheng 
et al., 2003). All of these studies indicate that SIRT1 is associated with cardiac function. Our study is the first to directly observe the heart function of SIRT1(+/-) mice in vivo. Moreover, we found that AMPK expression in the heart was lower in SIRT1(+/-) mice than in WT mice. Our results suggest that SIRT1 may be involved in the maintenance of the heart function and can regulate the expression of AMPK in mice hearts.

Previous in vitro studies suggested that resveratrol-induced cell survival was mediated by AMPK in $\mathrm{H} 9 \mathrm{c} 2$ cells, and resveratrol-induced glucose uptake was observed in vitro in H9c2 cardiac myoblast cells through the AMPK pathway (Hwang et al., 2008; Penumathsa et al., 2008). In our study, we also found that resveratrol, through SIRT1 activation, could increase AMPK expression in heart failure rats, and that the expression of AMPK was decreased in SIRT1(+/-) mice. All of these results indicate that SIRT1 can regulate the expression of AMPK. Therefore, in order to observe the effect of SIRT1 on AMPK expression, we cultivated an H9c2 cell line. In accordance with previous reports (Hwang et al., 2008; Penumathsa et al., 2008), we also found that resveratrol could increase the expression of AMPK. Because recent evidence indicated that resveratrol was not a direct activator of SIRT1 (Pacholec et al., 2010), we constructed the overexpression SIRT1 H9c2 cell line, and used nicotinamide to inhibit the activity of SIRT1. We found that SIRT1 could activate AMPK by itself. These results suggested that resveratrol likely activates AMPK by the SIRT1 pathway. Combining the results from heart failure rats and SIRT1(+/-) mice, we can conclude that resveratrol improves cardiac function by upregulating AMPK in heart failure.

It is reasonable to assume that the resveratrol-enriched diet was responsible for the lower mortality observed in developing post-myocardial infarction chronic heart failure. This may be due to its beneficial effects on heart failure or due to the activation of SIRT1. In the resveratrol group, SIRT1 expression was higher than that in the no-resveratrol group. Our data also showed that SIRT1(+/-) mice had shorter lifespans compared with WT mice. As SIRT1 was shown to slow the aging process and confer stress resistance to the heart in vivo (Alcendor et al., 2007), the reduced mortality of the resveratrol group observed in the present study was likely due to the higher expression of SIRT1. Therefore, we can conclude that resveratrol is a valuable drug for heart failure therapy.

Our study has some limitations. We could not perform Langendorff perfusing in SIRT1 knockout (-/-) mice, and there are several possible reasons to explain this. The initial investigations of Sirt1-deficient mice revealed a phenotype that included a reduced lifespan, small size, and an increased frequency of abnormal sperm (Cheng et al., 2003; Coussens et al., 2008). Second, Sirt1 deficiency was shown to markedly attenuate spermatogenesis (Coussens et al., 2008), making it difficult to breed healthy and adult mice for testing. Another study indicated that SIRT1 knockout (+/-) mice were useful for cardiac function investigations (Sulaiman et al., 2010).

In summary, our data presented here demonstrate that a natural substance (resveratrol) is capable of reversing the complications of heart failure by activating SIRT1-dependent transcriptional regulatory mechanisms, thereby restoring AMPK expression and reducing mortality. Our findings have broad implications because changes in AMPK expression levels are not limited to end-stage heart failure due to ischemic cardiomyopathy, but also play a role in hypertrophic cardiomyopathy (Turdi et al., 2011; Zhang et al., 2011b), diabetic hearts, and aging hearts, and resveratrol treatment has been found to be beneficial under all of these conditions (Thirunavukkarasu et al., 2007; Alcendor et al., 2007; Sulaiman et al., 2010). Therefore, our study might have profound implications for the treatment of heart failure and other cardiac disorders, and suggests that diets rich in resveratrol will prove beneficial in this disease. 


\title{
ACKNOWLEDGMENTS
}

\author{
Research supported by a grant from the National Natural Science Foundation of China \\ (grant \#30700380).
}

\section{Conflicts of interest}

The authors declare no conflict of interest.

\section{REFERENCES}

Alcendor RR, Kirshenbaum LA, Imai S, Vatner SF, et al. (2004). Silent information regulator 2alpha, a longevity factor and class III histone deacetylase, is an essential endogenous apoptosis inhibitor in cardiac myocytes. Circ. Res. 95: 971-980.

Alcendor RR, Gao S, Zhai P, Zablocki D, et al. (2007). Sirt1 regulates aging and resistance to oxidative stress in the heart. Circ. Res. 100: 1512-1521.

Beauloye C, Bertrand L, Horman S and Hue L (2011). AMPK activation, a preventive therapeutic target in the transition from cardiac injury to heart failure. Cardiovasc. Res. 90: 224-233.

Behbahani J, Thandapilly SJ, Louis XL, Huang Y, et al. (2010). Resveratrol and small artery compliance and remodeling in the spontaneously hypertensive rat. Am. J. Hypertens. 23: 1273-1278.

Cheng HL, Mostoslavsky R, Saito S, Manis JP, et al. (2003). Developmental defects and p53 hyperacetylation in Sir2 homolog (SIRT1)-deficient mice. Proc. Natl. Acad. Sci. U. S. A. 100: 10794-10799.

Cho IR, Koh SS, Malilas W, Srisuttee R, et al. (2012). SIRT1 inhibits proliferation of pancreatic cancer cells expressing pancreatic adenocarcinoma up-regulated factor (PAUF), a novel oncogene, by suppression of beta-catenin. Biochem. Biophys. Res. Commun. 423: 270-275.

Coussens M, Maresh JG, Yanagimachi R, Maeda G, et al. (2008). Sirt1 deficiency attenuates spermatogenesis and germ cell function. PLoS One 3: e1571.

Dekkers DH, Bezstarosti K, Gurusamy N, Luijk K, et al. (2008). Identification by a differential proteomic approach of the induced stress and redox proteins by resveratrol in the normal and diabetic rat heart. J. Cell Mol. Med. 12: 1677-1689.

Ghanim H, Sia CL, Korzeniewski K, Lohano T, et al. (2011). A resveratrol and polyphenol preparation suppresses oxidative and inflammatory stress response to a high-fat, high-carbohydrate meal. J. Clin. Endocrinol. Metab. 96: 1409-1414.

Hung LM, Chen JK, Huang SS, Lee RS, et al. (2000). Cardioprotective effect of resveratrol, a natural antioxidant derived from grapes. Cardiovasc. Res. 47: 549-555.

Hwang JT, Kwon DY, Park OJ and Kim MS (2008). Resveratrol protects ROS-induced cell death by activating AMPK in H9c2 cardiac muscle cells. Genes Nutr. 2: 323-326.

Imai S, Armstrong CM, Kaeberlein M and Guarente L (2000). Transcriptional silencing and longevity protein Sir2 is an NAD-dependent histone deacetylase. Nature 403: 795-800.

Jiang S, Wang W, Miner J and Fromm M (2012). Cross regulation of sirtuin 1, AMPK, and PPARgamma in conjugated linoleic acid treated adipocytes. PLoS One 7: e48874.

Kim MS and Lee KU (2005). Role of hypothalamic 5'-AMP-activated protein kinase in the regulation of food intake and energy homeostasis. J. Mol. Med. 83: 514-520.

Lu D, Ma Y, Zhang W, Bao D, et al. (2012). Knockdown of cytochrome P450 2E1 inhibits oxidative stress and apoptosis in the $\mathrm{cTnT}(\mathrm{R} 141 \mathrm{~W})$ dilated cardiomyopathy transgenic mice. Hypertension 60: 81-89.

Pacholec M, Bleasdale JE, Chrunyk B, Cunningham D, et al. (2010). SRT1720, SRT2183, SRT1460, and resveratrol are not direct activators of SIRT1. J. Biol. Chem. 285: 8340-8351.

Penumathsa SV, Thirunavukkarasu M, Zhan L, Maulik G, et al. (2008). Resveratrol enhances GLUT-4 translocation to the caveolar lipid raft fractions through AMPK/Akt/eNOS signalling pathway in diabetic myocardium. J. Cell Mol. Med. 12: 2350-2361.

Ramamurthy S and Ronnett GV (2006). Developing a head for energy sensing: AMP-activated protein kinase as a multifunctional metabolic sensor in the brain. J. Physiol. 574: 85-93.

Rojas M, Meredith D, Kylander J, Barrick D, et al. (2008). Echocardiography under isoflurane anesthesia affects heart rate and function in commonly utilized mouse strains. FASEB 22: 970-944. 
Shin JA, Lee KE, Kim HS and Park EM (2012). Acute resveratrol treatment modulates multiple signaling pathways in the ischemic brain. Neurochem. Res. 37: 2686-2696.

Sulaiman M, Matta MJ, Sunderesan NR, Gupta MP, et al. (2010). Resveratrol, an activator of SIRT1, upregulates sarcoplasmic calcium ATPase and improves cardiac function in diabetic cardiomyopathy. Am. J. Physiol. Heart Circ. Physiol. 298: H833-H843.

Thandapilly SJ, Wojciechowski P, Behbahani J, Louis XL, et al. (2010). Resveratrol prevents the development of pathological cardiac hypertrophy and contractile dysfunction in the SHR without lowering blood pressure. Am. J. Hypertens. 23: 192-196.

Thirunavukkarasu M, Penumathsa SV, Koneru S, Juhasz B, et al. (2007). Resveratrol alleviates cardiac dysfunction in streptozotocin-induced diabetes: Role of nitric oxide, thioredoxin, and heme oxygenase. Free Radic. Biol. Med. 43: 720-729.

Turdi S, Fan X, Li J, Zhao J, et al. (2010). AMP-activated protein kinase deficiency exacerbates aging-induced myocardial contractile dysfunction. Aging Cell 9: 592-606.

Turdi S, Kandadi MR, Zhao J, Huff AF, et al. (2011). Deficiency in AMP-activated protein kinase exaggerates high fat diet-induced cardiac hypertrophy and contractile dysfunction. J. Mol. Cell Cardiol. 50: 712-722.

Wang QD, Tokuno S, Valen G, Sjoquist PO, et al. (2002). Cyclic fluctuations in the cardiac performance of the isolated Langendorff-perfused mouse heart: pyruvate abolishes the fluctuations and has an anti-ischaemic effect. Acta Physiol. Scand. 175: 279-287.

Wojciechowski P, Juric D, Louis XL, Thandapilly SJ, et al. (2010). Resveratrol arrests and regresses the development of pressure overload- but not volume overload-induced cardiac hypertrophy in rats. J. Nutr. 140: 962-968.

Zaha VG and Young LH (2012). AMP-activated protein kinase regulation and biological actions in the heart. Circ. Res. 111: 800-814.

Zhang C, Feng Y, Qu S, Wei X, et al. (2011a). Resveratrol attenuates doxorubicin-induced cardiomyocyte apoptosis in mice through SIRT1-mediated deacetylation of p53. Cardiovasc. Res. 90: 538-545.

Zhang CX, Pan SN, Meng RS, Peng CQ, et al. (2011b). Metformin attenuates ventricular hypertrophy by activating the AMP-activated protein kinase-endothelial nitric oxide synthase pathway in rats. Clin. Exp. Pharmacol. Physiol. 38: 55-62. 